Manuelle Medizin 2008 · 46:336-336 DOI 10.1007/s00337-008-0641-2

Online publiziert: 21. September 2008

c) Springer Medizin Verlag 2008

M. Jensen Stochkendahl $l^{1}$ H.W. Christensen ${ }^{1} \cdot$ J. Hartvigsen ${ }^{1} \cdot$ W. Vach ${ }^{2} \cdot$ M. Haas ${ }^{3}$.

L. Hestbaek ${ }^{4} \cdot$ E. Adams ${ }^{5} \cdot$ G. Bronfort ${ }^{6}$

${ }^{1}$ Nordic Institute of Chiropractic and Clinical Biomechanics,

Part of Clinical Locomotion Science, Odense M

${ }^{2}$ The Department of Statistics, University of Southern Denmark, Odense

${ }^{3}$ Center for Outcomes Studies, Western States Chiropractic College, Portland

${ }^{4}$ The Back Research Center, Backcenter Funen; and Part of Clinical Locomotion Science, University of Southern Denmark, Odense

${ }^{5}$ Texas Chiropractic College, Pasadena

${ }^{6}$ Department of Research, Wolfe-Harris Center for Clinical Studies, Northwestern Health Sciences University, Bloomington

\title{
Manuelle Untersuchung der Wirbelsäule
}

\section{Manuelle Medizin (2007) 45: 301-308}

Folgende Punkte zu dem oben genannten Beitrag bedürfen der Richtigstellung:

- Der Verlag entschuldigt sich bei den Autoren dafür, dass für die Übersetzung Ihrer Arbeit und die Zweitpublikation keine Genehmigung eingeholt worden ist. Umso mehr danken wir für die nachträgliche Genehmigung zur Übersetzung und zum Nachdruck.

- Herr Prof. L. Beyer ist kein Mitautor und wurde vom Verlag versehentlich bei den Autoren der deutschsprachigen Publikation aufgeführt.

- Abbildungen, eine Tabelle, Anhänge sowie das Literaturverzeichnis des Originalbeitrags wurde in der Übersetzung fälschlicher Weise nicht wiedergegeben.

Die Originalpublikation Stochkendahl, MJ, Christensen HW, Hartvigsen J et al. (2006) Manual examination of the spine: a systematic critical literature review of reproducibility. J Manipulative Physiol Ther 29: 475-485 finden Sie zum Nachlesen unter folgender URL:

http://www.jmptonline.org/article/ So161-4754(06)oo155-2/fulltext
Wir bitten die Autoren der Originalpublikation und unsere Leserinnen und Leser, diese Fehler zu entschuldigen.

\section{Korrespondenzadresse}

M. Jensen Stochkendahl

Nordic Institute of Chiropractic and Clinical Biomechanics, Part of Clinical Locomotion Science Forskerparken 10A, 5230 Odense M

Dänemark

m.jensen@nikkb.dk
Die Online-Version des Originalartikels können Sie unter http://dx.doi.org/10.1007/s00337-0070548-3 finden. 